12

\title{
Эффективный ротационный потенциал молекулярных ионов в плоской радиочастотной ловушке
}

\author{
(С) И.А. Васильев, ${ }^{1}$ О.М. Кущенко, ${ }^{1}$ С.С. Рудый, ${ }^{1,2,9}$ Ю.В. Рождественский ${ }^{1}$ \\ ${ }^{1}$ Санкт-Петербургский национальный исследовательский университет \\ информационных технологий, механики и оптики (Университет ИТМО), \\ 197101 Санкт-Петербург, Россия \\ ${ }^{2}$ OОО „КОМПЕТЕНТУМ.РУ“, \\ 141700 Долгопрудный, Московская обл., Россия \\ ฯ e-mail:semyonrudiy@gmail.com
}

Поступило в Редакцию 5 декабря 2018 г.

В окончательной редакции 8 февраля 2019 г. Принято к публикации 19 марта 2019 г.

\begin{abstract}
Впервые рассмотрена модель эффективного ротационного потенциала, который выступает аналогом псевдопотенциала в случае локализации модели двухатомной частицы с жесткой связью в плоской квадрупольной радиочастотной ловушке. Было продемонстрировано, что эффективный ротационный потенциал может использоваться для описания динамики широкого диапазона двухатомных частиц с различными центрами масс и зарядов. Сравнивая модель псевдопотенциала для локализации одиночного иона и настоящую модель эффективного ротационного потенциала для двухатомной структуры, были определены дополнительные положения квазиравновесия как для центра масс двухатомной частицы, так и для угла ориентации молекулы. Дополнительные положения квазиравновесия значительно влияют на динамику двухатомной заряженной структуры при локализации в поле плоской квадрупольной ионной ловушки.
\end{abstract}

Ключевые слова: эффективный потенциал, псевдопотенциал, ионная ловушка, молекулярная масс-спектрометрия, методы усреднений.

DOI: $10.21883 /$ JTF.2019.09.48074.422-18

\section{Введение}

На сегодняшний день проблема локализации молекулярных ионов и других заряженных частиц представляет большой интерес [1-5]. Настоящий интерес, прежде всего, вызван необходимостью устойчивой локализации молекулярных ионов и сложных биомолекулярных комплексов в различных приложениях масс-спектрометрии, медицины и биофизики. Тем не менее, локализация и манипулирование сложными заряженными объектами сопряжена с большим количеством проблем, поскольку динамика пространственно-неоднородных заряженных частиц значительно зависит от пространственной структуры таких объектов [6]. Таким образом, даже одна дополнительная вращательная степень свободы в сравнении со случаем локализации одноатомной молекулы значительно усложняет движение частицы в пространстве, приводя к изменению частотных спектров (в классическом рассмотрении), энергетических уровней (в квантово-механическом рассмотрении), что, к примеру, исключает возможность эффективного лазерного охлаждения [7].

Хорошо известно, локализация одноатомных ионов в квадрупольной ионной ловушке описывается моделью псевдопотенциала. Впоследствии, идеи эффективной потенциальной функции в квадрупольной ловушке получили свое развитие на случаи мультипольных ионных ловушек, локализации в фазовой и частотной модуляции и другие [8]. Тем не менее существующие модели эффективного потенциала не могут быть применены для анализа динамики „двухатомной“ заряженной структуры, поскольку в случае локализации таких частиц в квадрупольной ловушке, уравнения движения и центра масс, и угловой ориентации существенно нелинейны (по сравнению с уравнениями движения для одиноатомного иона) из-за возникающей связи пространственного положения иона с его угловой ориентацией. Однако существующий метод полного разделения движения хорошо подходит для описания динамики в быстро осциллирующих полях $[8,9]$. В частности, настоящий метод также применим и для анализа движения одиночного иона в квадрупольных и мультипольных радиочастотных ловушках [12].

В настоящей работе мы впервые представим модифицированные уравнения движения двухатомного молекулярного иона, полученные методом полного разделения движения. В результате проведенного анализа, в настоящей работе представлен аналог псевдопотенциала для двухатомной заряженной частицы - эффективный ротационный потенциал. Полученный эффективный ротационный потенциал позволяет описывать дополнительные положения квазиравновесия, которые возникают из-за нелинейной динамики частицы со сложной пространственной структурой. Таким образом, эффективный ротационный потенциал является универсальной моделью описания движения заряженных двухатомных частиц, 
поскольку усредненные уравнения движения не зависят от времени в явном виде и поддаются аналитическому исследованию, позволяя получить более детальное представление о динамике двухатомной частицы. Предложенная модель позволяет прогнозировать частотные спектры вращения, которые зависят от параметров частицы (масс и зарядов), а также от начальных условий скорости и инжекции по обобщенным координатам. Наконец, аналитические выражения для эффективного ротационного потенциала открывают новые возможности для управления динамикой заряженных биомолекулярных структур, решения задач детектирования хиральности заряженных частиц, а также реализации ротационного охлаждения двухатомных молекулярных ионов.

Настоящая работа состоит из трех разделов и заключения. В первом разделе рассмотрен физический принцип локализации двухатомного иона с жесткой связью в квадрупольной ионной ловушке. Во втором разделе приведено усреднение уравнений движения с последующим выводом эффективного ротационного потенциала. Третий раздел посвящен проблеме локализации двухатомного иона в частных случаях - радиально симметричной и асимметричной молекул. Произведен расчет положений квазиравновесия для различных случаев локализации, и рассмотрено влияние формы эффективного потенциала на динамику иона.

\section{1. Физический принцип локализации}

Рассмотрим локализацию двухатомной заряженной частицы с жесткой связью в квадрупольной радиочастотной ионной ловушке (рис. 1).

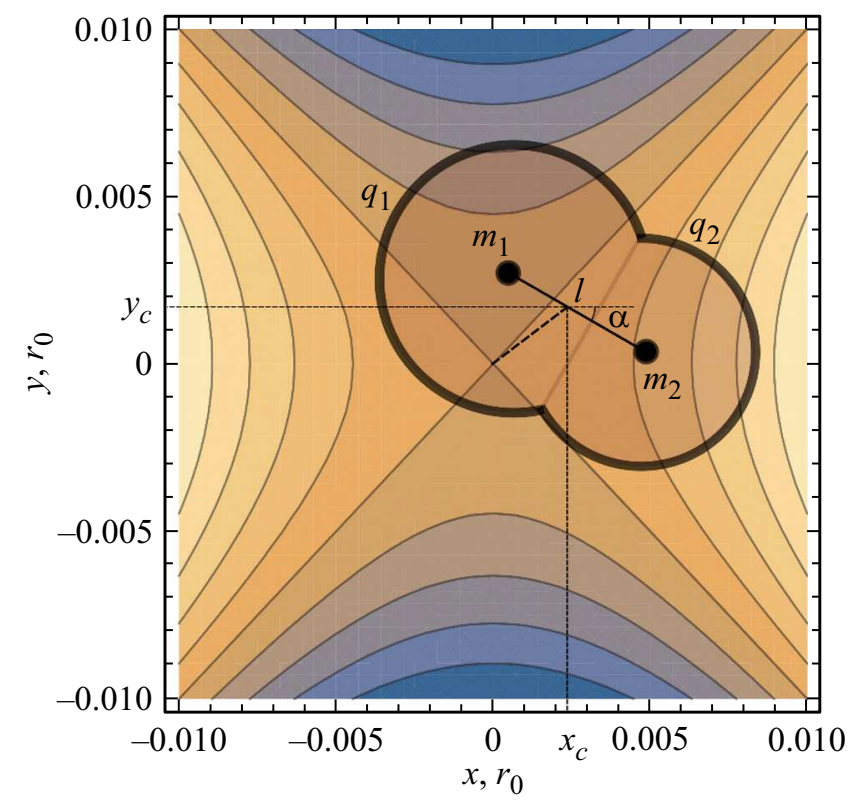

Рис. 1. Модель жесткого двухатомного молекулярного иона с массами $\left(m_{1}, m_{2}\right)$ и зарядами $\left(q_{1}, q_{2}\right)$ в линейной радиочастотной квадрупольной ловушке без компоненты постоянного поля.
Отметим, что термин „двухатомныйс охватывает широкий спектр различных заряженных структур, включающих как микро-, так и наноструктуры с разделенными центрами массы и заряда. Предлагаемая „двухатомная“ модель может быть использована для описания движения двухатомных молекулярных ионов, нанотрубок, простых заряженных биологических объектов и др. [10-13].

Далее декартовы координаты компонент двухатомной заряженной частицы $\left(m_{1}, q_{1}\right)$ и $\left(m_{2}, q_{2}\right)$ выражены через обобщенные координаты движения центра масс $\left(x_{c}, y_{c}\right)$, угловой ориентации $(\alpha)$ и межатомарного расстояния $2 l$.

$$
\begin{aligned}
& x_{1}=x_{c}-\frac{l m_{2} \cos \alpha}{m_{1}+m_{2}}, \quad y_{1}=y_{c}-\frac{l m_{2} \sin \alpha}{m_{1}+m_{2}}, \\
& x_{2}=x_{c}+\frac{l m_{1} \cos \alpha}{m_{1}+m_{2}}, \quad y_{2}=y_{c}+\frac{l m_{1} \sin \alpha}{m_{1}+m_{2}} .
\end{aligned}
$$

Лагранжиан динамической системы, описывающий движение заряженной двухатомной молекулы в поле квадрупольной ионной ловушки, примет вид

$$
\begin{aligned}
& L=\frac{m_{1}+m_{2}}{2}\left(\dot{x}_{c}^{2}+\dot{y}_{c}^{2}\right)+\frac{m_{1} m_{2} l^{2} \dot{\alpha}^{2}}{2\left(m_{1}+m_{2}\right)}-\frac{V \cos (\omega t)}{r_{0}^{2}} \\
& \times\left[\left(x_{c}^{2}-y_{c}^{2}\right)\left(q_{1}+q_{2}\right)+\frac{2 l\left(m_{1} q_{2}-m_{2} q_{1}\right)}{m_{1}+m_{2}}\right. \\
& \left.\times\left(x_{c} \cos (\alpha)-y_{c} \sin (\alpha)\right)+\frac{\left(m_{1}^{2} q_{2}-m_{2}^{2} q_{1}\right) l^{2}}{\left(m_{1}+m_{2}\right)^{2}} \cos (2 \alpha)\right],
\end{aligned}
$$

где $V[\mathrm{~V}]$ - амплитуда переменного напряжения, приложенного к электродам ловушки, $r_{0}[\mathrm{~cm}]$ - радиус ловушки, $\omega[\mathrm{rad} / \mathrm{s}]$ - частота радиочастотного электрического поля, $t[\mathbf{s}]$ - время. Вводя параметры вида

$$
\begin{aligned}
& \beta_{1}=\frac{2 V\left(q_{1}+q_{2}\right)}{r_{0}^{2} \omega^{2}\left(m_{1}+m_{2}\right)}, \quad \beta_{4}=\frac{2 V\left(m_{1}^{2} q_{2}-m_{2}^{2} q_{1}\right)}{r_{0}^{2} \omega^{2} m_{1} m_{2}\left(m_{1}+m_{2}\right)}, \\
& \beta_{2}=\frac{2 V\left(m_{1} q_{2}-m_{2} q_{1}\right)}{r_{0}^{2} \omega^{2}\left(m_{1}+m_{2}\right)}, \quad \beta_{3}=\frac{2 V\left(m_{1} q_{2}-m_{2} q_{1}\right)}{r_{0}^{2} \omega^{2} m_{1} m_{2}},
\end{aligned}
$$

а также $\tau=\omega t$ уравнения движения в координатах „центр масс-угол“ и введенных выше параметрах примут вид

$$
\begin{gathered}
\ddot{x}+\cos (2 \tau)\left(\beta_{1} x+\beta_{2} \cos (\alpha)\right)=0, \\
\ddot{y}-\cos (2 \tau)\left(\beta_{1} y+\beta_{2} \sin (\alpha)\right)=0, \\
\ddot{\alpha}-\cos (2 \tau)\left[\beta_{3}(x \sin (\alpha)+y \cos (\alpha))+\beta_{4} \sin (2 \alpha)\right]=0 .
\end{gathered}
$$

Введенные коэффициенты $\beta_{2}$ и $\beta_{3}$ связывают уравнения (6)-(8), определяя нелинейный тип движения заряженных частиц (мы будем называть $\beta_{2}$ и $\beta_{3}$,кроссконстантами“'). Между тем коэффициенты $\beta_{1,4}$ являются „Линейными“ коэффициентами, поскольку они характеризуют независимое движение по соответствующим автономным степеням свободы. Можно видеть, что коэффициент $\beta_{1}$ характеризует движение центра масс, 
a $\beta_{4}$ определяет угол пространственной ориентации. Отметим, что все коэффициенты $\beta_{1-4}$ зависят как от характеристик ловушки, так и от отношения зарядов/масс, что приводит к сильному влиянию пространственной структуры заряженной двухатомной частицы на динамику системы и процесс локализации в целом.

\section{2. Эффективный ротационный потенциал}

Псевдопотенциал для случая локализации одноатомного одиночного иона в квадрупольной ионной ловушке принимает вид

$$
U_{\text {pseudo }}(x, y)=\frac{1}{4} \frac{\left(q_{0} V\right)^{2}}{m \omega^{2} r_{0}^{2}}\left(x^{2}+y^{2}\right),
$$

где $m$ и $q_{0}$ - масса и заряд одиночного иона. Представленный псевдопотенциал для случая локализации одноатомного иона характеризуется квадратичной зависимостью от координат только при гиперболической форме электродов ионной ловушки, отсутствие влияния мультипольного РЧ-потенциала и прочих негативных эффектов, влияющих на динамику иона. В случае двухатомной заряженной частицы дополнительная степень свободы существенно модифицирует характер движения частицы, даже при идеальной конфигурации ловушки. Таким образом, изменение пространственной структуры локализованного объекта приводит к существенному изменению эффективной потенциальной функции. Как видно, уравнения движения двухатомной заряженной структуры в квадрупольной ионной ловушке содержат быстро осциллирующие гармонические члены, явно зависящие от времени (6)-(8). Уравнения движения (6)-(8) обычно решаются численными методами расчета траекторий ионов методом Рунге-Кутта для частных случаев начальных условий инжекции, не давая полного представления о динамике частицы. В то же время концепция аналитического псевдопотенциала (9) не может быть использована для случая двухатомных заряженных частиц. Однако исследование движения заряженных частиц аналитическими методами становится возможным после усреднения оригинальных уравнений движения методом полного разделения движения в виде

$$
\begin{gathered}
x=X\left(\bar{X}, f\left(\epsilon_{x}\right)\right)+\epsilon_{x}, \quad y=Y\left(\bar{Y}, f\left(\epsilon_{y}\right)\right)+\epsilon_{y}, \\
\alpha=\psi\left(\bar{\psi}, f\left(\epsilon_{\psi}\right)\right)+\epsilon_{\alpha} .
\end{gathered}
$$

Полное секулярное движение захваченной заряженной частицы $(X, Y, \psi)$ описывается совокупностью „медленной“ составляющей движения за счет взаимодействия с постоянной компонентой электрического поля $(\bar{X}, \bar{Y}, \bar{\psi})$ и дополнительной силы, индуцированной влиянием быстроосциллирующих компонент $(f(\epsilon(x, y, \psi)))$ (аналог „псевдопотенциала“ (9)). В случае локализации заряженной частицы в электрическое поле без постоянной компоненты напряжения на электродах, члены $(\bar{X}, \bar{Y}, \bar{\psi})$ равны нулю. Таким образом, секулярное движение двухатомной заряженной частицы определяется только результирующей „быстрых“ осцилляций $(f(\epsilon(x, y, \psi)))$, эффективный потенциал которой определяется полной кинетической энергией (10) вида

$$
\Phi=\frac{1}{2 \pi} \sum_{i=1}^{3} \int_{0}^{2 \pi} \frac{\left(\epsilon_{i}^{\prime}(Q, \tau)\right)^{2}}{2} d \tau,
$$

где $Q$ - обобщенные координаты (см. Приложение). Подставив обобщенные кооринаты „центр масс-угол“, эффективная потенциальная энергия примет вид

$$
\begin{aligned}
& \Phi=\frac{1}{16}\left[\left(\beta_{1} X+\beta_{2} \cos (\psi)^{2}\right)+\left(\beta_{1} Y+\beta_{2} \sin (\psi)^{2}\right)\right. \\
& \left.+\left(\beta_{3}(X \sin (\psi)+Y \cos (\psi))+\beta_{4} \sin (2 \psi)\right)^{2}\right] .
\end{aligned}
$$

Сложная форма усредненной потенциальной функции, описываемая уравнением (12), существенно зависит от усредненной угловой ориентации иона в пространстве $\psi$. В силу периодичности $\psi$-зависимых членов, пространственное распределение такого потенциала также имеет $\psi$-периодическую структуру. В общем случае, мы можем представить эффективный ротационный потенциал как сумму независимых компонент:

$$
\begin{gathered}
\Phi=\Phi_{\text {lin }}+\Phi_{\text {rot }}+\Phi_{\text {cross }}, \\
\Phi_{\text {lin }}=\frac{1}{16}\left(X^{2}+Y^{2}\right) \beta_{1}^{2}, \\
\Phi_{\text {rot }}=\frac{1}{16}(\sin (2 \psi)) \beta_{4}^{2}, \\
\Phi_{\text {cross }}=\frac{1}{8}(X \cos (\psi)+Y \sin (\psi)) \beta_{1} \beta_{2} \\
+\frac{1}{8}\left(\beta_{3} \beta_{4}(X \sin (\psi)+Y \cos (\psi)) \sin (2 \psi)\right. \\
+\frac{\beta_{3}^{2}}{16}(Y \cos (\psi)+X \sin (\psi))^{2}+\frac{1}{16} \beta_{2}^{2} .
\end{gathered}
$$

Уравнение (14) описывает ,линейную“ компоненту, которая определяет линейно возвращающую силу для центра масс двухатомной заряженной частицы в ионной ловушке, и $\Phi_{l i n}$ полностью совпадает с псевдопотенциалом для одиночного иона (9). Выражение для $\Phi_{\text {rot }}(15)-\psi$-периодическая составляющая, обусловленная „медленными“ колебаниями ориентации иона в простанстве (рис. 1); $\Phi_{\text {cross }}(16)$ - член, описывающий смешанные компоненты $\Phi_{l i n}$ и $\Phi_{\text {rot }}$ („кросспсевдопотенциал“ ). Таким образом, псевдопотенциал одиночного иона (9) лишь частично описывает движение двухатомных частиц в квадрупольной ионной ловушке, соответсвуя линейной компоненте $\Phi_{\text {lin }}$.

В то же время $\Phi_{l i n}$ и $\Phi_{\text {rot }}$ зависят только от линейных констант $\beta_{1}, \beta_{4}$, когда $\Phi_{\text {cross }}$ зависит от линейных и 
кросс-констант $\beta_{2}, \beta_{3}$. Значения различных компонент эффективного ротационного потенциала (14), (15) также зависит от конфигурации ловушки и характеристик заряженной частицы (массы, заряды и межатомного расстояния). Таким образом, мы можем наблюдать значительные изменения усредненной динамической системы по сравнению с оригинальной динамикой одиночного иона. В результате проведения операции усреднения результирующие уравнения движения определяются пространственными производными потенциала (12) по соответствующим обобщенным координатам в виде

$$
\begin{aligned}
\ddot{X}= & -\frac{1}{8}\left[\beta_{1}^{2} X+\beta_{1} \beta_{2} \cos (\psi)+\beta_{3} \sin (\psi)\left(\beta_{3}(X \sin (\psi)\right.\right. \\
& \left.\left.+Y \cos (\psi))+\beta_{4} \sin (2 \psi)\right)\right], \\
\ddot{Y}= & -\frac{1}{8}\left[\beta_{1}^{2} Y+\beta_{1} \beta_{2} \sin (\psi)+\beta_{3} \cos (\psi)\left(\beta_{3}(X \sin (\psi)\right.\right. \\
& \left.\left.+Y \cos (\psi))+\beta_{4} \sin (2 \psi)\right)\right], \\
\ddot{\psi}= & -\frac{1}{8}\left[-\beta_{2} \sin (\psi)\left(\beta_{1} X+\beta_{2} \cos (\psi)\right)+\beta_{2} \cos (\psi)\right. \\
& \times\left(\beta_{1} Y+\beta_{2} \sin (\psi)\right)+\left(2 \beta_{4} \cos (2 \psi)\right. \\
& \left.+\beta_{3}(X \cos (\psi)-Y \cos (\psi))\right)\left(\beta_{3}(X \sin (\psi)\right. \\
& \left.\left.+Y \cos (\psi))+\beta_{4} \sin (2 \psi)\right)\right] .
\end{aligned}
$$

Уравнения движения (17)-(19) являются автономными и полностью определяют секулярную динамику двухатомных заряженных частиц в ионной ловушке. В общем случае, эффективный ротационный потенциал определяется девятью дополнительными локальными потенциальными минимумами по сравнению с локализацией одиночного иона. Пространственные координаты положений квазиравновесия примут вид

$$
\begin{gathered}
\tilde{X}_{1}=0, \quad \tilde{Y}_{1}=-\frac{\beta_{2}}{\beta_{1}}, \quad \tilde{\psi}_{1}=\frac{\pi}{2}+2 \pi k, \\
\tilde{X}_{2}=0, \quad \tilde{Y}_{2}=\frac{\beta_{2}}{\beta_{1}}, \quad \tilde{\psi}_{2}=-\frac{\pi}{2}+2 \pi k, \\
\tilde{X}_{3}=-\frac{\beta_{2}}{\beta_{1}}, \quad \tilde{Y}_{3}=0, \quad \tilde{\psi}_{3}=2 \pi k, \\
\tilde{X}_{4}=\frac{\beta_{2}}{\beta_{1}}, \quad \tilde{Y}_{4}=0, \quad \tilde{\psi}_{4}=\pi+2 \pi k, \\
\tilde{X}_{5}=\frac{\beta_{1} \beta_{2}+\beta_{3} \beta_{4}}{\sqrt{2}\left(\beta_{1}^{2}+\beta_{3}^{2}\right)}, \quad \tilde{Y}_{5}=\frac{\beta_{1} \beta_{2}+\beta_{3} \beta_{4}}{\sqrt{2}\left(\beta_{1}^{2}+\beta_{3}^{2}\right)} \\
\tilde{\psi}_{5}=-\frac{3 \pi}{4} 2 \pi k, \\
\tilde{X}_{6}=\frac{\beta_{1} \beta_{2}+\beta_{3} \beta_{4}}{\sqrt{2}\left(\beta_{1}^{2}+\beta_{3}^{2}\right)}, \quad \tilde{Y}_{6}=-\frac{\beta_{1} \beta_{2}+\beta_{3} \beta_{4}}{\sqrt{2}\left(\beta_{1}^{2}+\beta_{3}^{2}\right)} \\
\tilde{\psi}_{6}=\frac{3 \pi}{4}+2 \pi k,
\end{gathered}
$$

$$
\begin{gathered}
\tilde{X}_{7}=-\frac{\beta_{1} \beta_{2}+\beta_{3} \beta_{4}}{\sqrt{2}\left(\beta_{1}^{2}+\beta_{3}^{2}\right)}, \quad \tilde{Y}_{7}=\frac{\beta_{1} \beta_{2}+\beta_{3} \beta_{4}}{\sqrt{2}\left(\beta_{1}^{2}+\beta_{3}^{2}\right)}, \\
\tilde{\psi}_{7}=-\frac{\pi}{4}+2 \pi k \\
\tilde{X}_{8}=-\frac{\beta_{1} \beta_{2}+\beta_{3} \beta_{4}}{\sqrt{2}\left(\beta_{1}^{2}+\beta_{3}^{2}\right)}, \quad \tilde{Y}_{8}=-\frac{\beta_{1} \beta_{2}+\beta_{3} \beta_{4}}{\sqrt{2}\left(\beta_{1}^{2}+\beta_{3}^{2}\right)}, \\
\tilde{\psi}_{8}=\frac{\pi}{4}+2 \pi k .
\end{gathered}
$$

В результате динамическая система, описываемая потенциальной функцией (12) имеет восемь дополнительных положений квазиравновесия с четырьмя устойчивыми положениями (20)-(23). Ниже более подробно рассматривается влияние формы эффективного ротационного потенциала на движение двухатомной заряженной структуры.

\section{3. Результаты и обсуждение}

\section{1. Эффективный ротационный потенциал радиально-симметричной молекулы}

Рассмотрим простейшую форму эффективного ротационного потенциала для случая симметричной модели двухатомного иона с $m_{1}=m_{2}$ и $q_{1}=q_{2}$. Кросс-константы $\beta_{2,3}$, определяющие нелинейный тип движения двухатомных частиц, равны нулю. В таком случае как исходные уравнения движения (6)-(8), так и усредненные уравнения движения (17)-(19) полностью разделяются на движение центра масс и изменение соответствующего угла ориентации. Строго говоря, исходные уравнения движения центра масс для симметричной

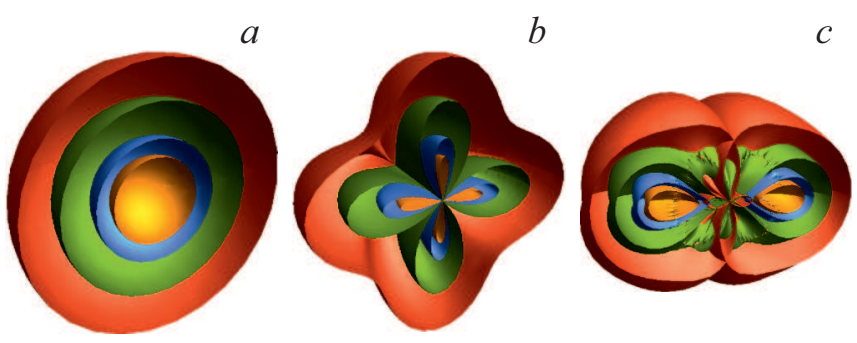

Рис. 2. Эквипотенциальные поверхности псевдопотенциала $(a)$ (9) и эффективного ротационного потенциала $(b, c)$ (уравнение (11)) в полярных координатах (см. Приложение): $a$ - эквипотенциальные поверхности эффективного потенциала для одиночного иона в квадрупольной ионной ловушке [12] в полярных координатах. Отчетливо наблюдается, что динамическая система (17-19) имеет единственную точку квазиравновесия в геометрическом центре ловушки; $b-$ эффективный ротационный потенциал радиально-симметричной молекулы. Глубина эффективного ротационного потенциала зависит от значения линейных $\beta_{1,4}$-констант, реализуется четыре положения квазиравновесия в геометрическом центре ловушки с различной угловой ориентацией; $c-$ эффективный ротационный потенциал асимметричной молекулы, глубина и пространственное положение дополнительных точек квазиравновесия зависят от значений кросс-констант $\beta_{2,3}$. 
a

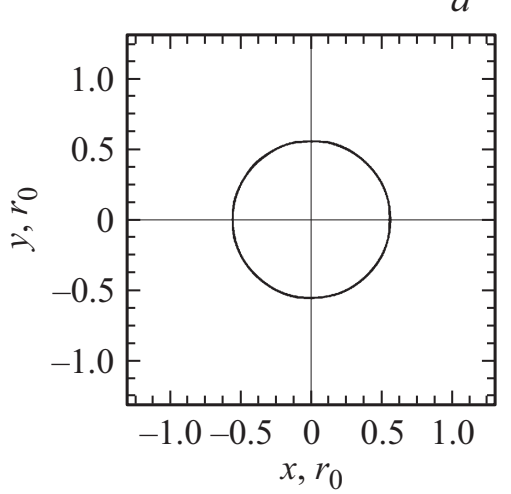

$b$

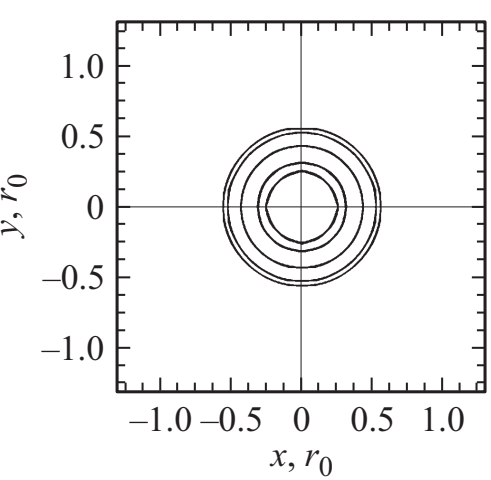

C

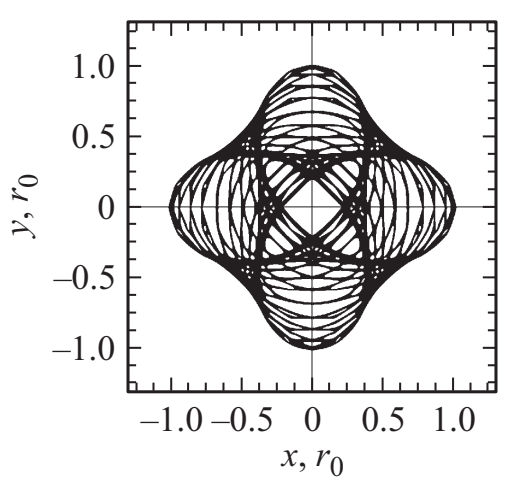

Рис. 3. Стробоскопическая секция псевдопотенциала (9) и ротационного потенциала (11) с шагом $\delta \psi=\pi / 16$ в плоскости $X O Y$. $a$ - соответствует стробоскопическому сечению псевдопотенциала, $b$ - сечению ротационного потенциала радиальносимметричной молекулы, $c$ - сечению ротационного потенциала асимметричной молекулы. Предложенные сечения соответствуют эквипотенциальным поверхностям рис. 2.

двухатомной частицы принимают форму уравнений Матье с нулевой $\tau$-независимой компонентой. Полное секулярное движение частицы в плоскости $X Y$ описывается только линейно-возвращающей силой. Можно заметить, что усредненные уравнения движения центра масс для симметричной двухатомной частицы без $\psi$-компоненты хорошо согласуются с классическим псевдопотенциалом (9) и соответствуют локализации одиночного иона в квадрупольной ионной ловушке (рис. 2,a). Тем не менее полный набор уравнений движения для симметричной заряженной частицы включает компоненту вращения $\psi$, характеризуя, таким образом, четыре $\psi$-зависящих локальных минимума. Такая динамическая система характеризуется четырьмя устойчивыми квазиравновесными точками, каждая из которых находится в геометрическом центре ловушки $\left(\tilde{X}_{i}=\tilde{Y}_{i}=0\right)$ и имеет разный ориентационный угол по координате $\psi\left(\tilde{\psi}_{i}=\frac{\pi}{2} k\right.$, $k \in Z)$ (20)-(23). На рис. 2, $b$ представлены эквипотенциальные поверхности эффективного ротационного потенциала в полярных координатах усредненной системы для симметричной двухатомной заряженной частицы. В случае инжекции заряженной частицы в одно из устойчивых положений квазиравновесия (20)-(23) частица сохраняет ориентацию и колеблется вблизи квазиравновесной точки с амплитудой $\Delta \psi<\psi / 8$. Подобный характер локализации может быть реализован только при низкой начальной скорости инжекции, что соответствует начальной кинетической энергии, не превышающей максимальную глубину потенциальной ямы в $\psi$-плоскости. Тем не менее, как уже отмечалось выше, независимо от вращения по углу ориентации движение двухатомной заряженной частицы с радиальной симметрией факторизуется, и мы можем анализировать динамику центра масс двухатомного молекулярного иона, аналогично одноатомному иону согласно классической теории псевдопотенциала.

\section{2. Эффективный ротационный потенциал асимметричной молекулы}

Зачастую заряженные диатомные частицы имеют асимметричную структуру, такие, как, например, наночастицы, простую, как биологические объекты (клетки, тромбоциты) и другие. В этом случае эффективный ротационный потенциал должен принимать более сложную форму, допускающую одновременное существование всех дополнительных потенциальных минимумов (20)-(27). На рис. 2, с представлено сечение эквипотенциальных поверхностей эффективного ротационного потенциала для значения параметров $\beta_{1,4}=1, \beta_{2,3}=0.3$. Отметим, что устойчивые положения квазиравновесия динамической системы (6)-(19) существенно зависят от значения параметров $\beta_{1,2}$. Для более глубокого анализа рассмотрим стробоскопическое сечение [14] эквипотенциальных поверхностей для анализа влияния потенциальных минимумов эффективного ротационного потенциала в случае асимметричной двухатомной частицы. Известно, что стробоскопическое сечение эквипотенциальной поверхности может быть построено как суперпозиция двумерных сечений в плоскости $\psi \mathrm{c}$ дискретным шагом $\Delta \psi$ (без потери общности решения, в настоящей работе рассматривалось сечение с шагом $\left.\Delta \psi=\frac{\pi}{16}\right)$ в плоскости координат центра масс усредненного движения.

На рис. 3 приведено стробоскопическое сечение эквипотенциальных поверхностей для различных значений параметров $\beta$. На рис. 3, $a$ представлено стробоскопическое сечение псевдопотенциала для одноатомного иона (9), который соответствует эффективному ротационному потенциалу для значений параметров $\beta_{2,3,4}=0$, $\beta_{1}>0$. В этом случае стробоскопическое сечение имеет круглую форму, поскольку псевдопотенциал для одиночного иона в квадрупольной ионной ловушке независим от значения угловой ориентации. На рис. $3, b$ представлено стробоскопическое сечение для значений 
параметров $\beta_{2,3}=0, \beta_{1,3}>0$. Этот случай соответствует эффективному ротационному потенциалу симметричной заряженной двухатомной структуре. Поскольку эффективный потенциал в таком случае характеризуется разделенными линейными и $\psi$-периодическими компонентами $\Phi_{\text {lin }}$ и $\Phi_{\text {rot }}$, можно видеть, что значение линейных констант $\beta_{1,4}$ отвечает только за глубину эффективного потенциала в зависимости от угла ориентации.

В то же время, отличное от нуля значение кроссконстант (5) значительно меняет форму эффективного потенциала. Увеличение кросс-константы $\beta_{2}$ приводит к возникновению дополнительных потенциальных минимумов (согласно (20)-(23)) (рис. 2, $b$ и 3, b). Эквипотенциальная кривая стробоскопического сечения принимает форму гипотрохоиды или эпитрохоиды, форма которой определяется численным значением кросс-констант. Наиболее сложная форма потенциала с ненулевыми кросс-константами характеризуется гипотрохоидоподобной кривой, которая описывается движением неподвижной точки на эллипсе переменного радиуса (рис. 3, $c$ ).

Такая форма (на рис. 3,c) соответствует случаю локализации частицы с асимметричной структурой. Пространственно эффективный ротационный потенциал для асимметричных заряженных двухатомных частиц выглядит как гофрированный цилиндр в декартовых координатах, изогнутый так, что трохоидоподобная кривая имеет форму с четырьмя „лепестками“ (рис. 3,c). Устойчивые положения квазиравновесия динамической системы, описываемые общей формой (12), определяются центрами соответствующих ,лепестков“ (вырожденный случай, где „лепестки“ отчетливо прослеживаются, представлен на рис. 2, $b$ (случай симметричной молекулы)). Однако локализация движения в координатах центра масс в устойчивых точках квазиравновесия в ,лепестках“ (20)-(23) сильно зависит от начальных условий инжекции как угловых, так и скоростей центра масс (что является прямым следствием нелинейной динамики локализованных частиц).

\section{Заключение}

Представленный выше эффективный ротационный потенциал является универсальной моделью, позволяющей изучать как простые одноатомные, так и сложные двухатомные микро- и макрочастицы. Пространственное положение заряженной частицы зависит от потенциального минимума эффективного ротационного потенциала и хорошо согласуется с оригинальными уравнениями движения (6)-(8). Отметим, что нелинейные эффекты, проявляющиеся в движении двухатомных частиц, характерны для двухатомной структуры с большим межатомным расстоянием, а также асимметричными полярными молекулами и диполями. В то же время даже для дипольных молекул, не имеющих непосредственного влияния электрического поля, ротационный потенциал характеризует линейно возвращающую силу, пропорциональную кросс-константе $\beta_{3}$. Было получено, что существующая модель псевдопотенциала описывает лишь линейную компоненту существующей эффективной потенциальной энергии. Предложенная модель эффективного ротационного потенциала открывает широкие возможности для анализа локализации двухатомных частиц.

\section{Финансирование работы}

Работа поддержана Министерством образования и науки РФ (проект 3.821.2014/K), грантом 074-U01 для лидирующих университетов РФ и грантом РФФИ 17-02-00598 A.

\section{Конфликт интересов}

Авторы заявляют, что у них нет конфликта интересов.

\section{Приложение}

\section{Уравнения эффективного ротационного потенциала в сферических координатах}

Рассмотрим координаты полного разделения движения, приведенные в (10)

$$
\begin{gathered}
x=X\left(\bar{X}, f\left(\epsilon_{x}\right)\right)+\epsilon_{x}, \quad y=Y\left(\bar{Y}, f\left(\epsilon_{y}\right)\right)+\epsilon_{y}, \\
\alpha=\psi\left(\bar{\psi}, f\left(\epsilon_{\psi}\right)\right)+\epsilon_{\alpha} .
\end{gathered}
$$

Как было отмечено выше, полное секулярное движение захваченной заряженной частицы $(X, Y, \psi)$ описывается совокупностью „медленной“ составляющей движения за счет взаимодействия с постоянной компонентой электрического поля $(\bar{X}, \bar{Y}, \bar{\psi})$ и дополнительной силы, индуцированной влиянием быстроосциллирующих компонент $(f(\epsilon(x, y, \psi)))$ (аналог ,псевдопотенциала“ (9)). В случае локализации заряженной частицы в электрическое поле без постоянной компоненты напряжения на электродах члены $(\bar{X}, \bar{Y}, \bar{\psi})$ равны нулю. Таким образом, секулярное движение двухатомной заряженной частицы определяется только результирующей „быстрых“ осцилляций $(f(\epsilon(x, y, \psi)))$. Подставляя введенные координаты (10) в оригинальные уравнения движения (6)-(8), определим новые уравнения движения. Без потери общности решения детально рассмотрим только уравнение движения по $O X$ вида

$$
\begin{aligned}
& \frac{d^{2}}{d \tau^{2}}\left(X\left(\bar{X}, f\left(\epsilon_{x}\right)\right)+\epsilon_{x}\right)+\cos (2 \tau)\left(\beta_{1}\left(X\left(\bar{X}, f\left(\epsilon_{x}\right)\right)+\epsilon_{x}\right)\right. \\
& \left.+\beta_{2} \cos \left(\psi\left(\bar{\psi}, f\left(\epsilon_{\psi}\right)\right)+\epsilon_{\alpha}\right)\right)=0
\end{aligned}
$$

Очевидно, что $\frac{d^{2}}{d \tau^{2}} X\left(\bar{X}, f\left(\epsilon_{x}\right)\right)$ не зависит от безразмерного времени в явном виде, а ускорение по быстроосциллирующим членам ввиду малости и отсутствия членов, характеризующихся постоянным напряжением $\bar{X}$, 
примет вид

$\frac{d^{2}}{d \tau^{2}} \epsilon_{x}=-\cos (2 \tau)\left(\beta_{1}\left(X\left(f\left(\epsilon_{x}\right)\right)+\beta_{2} \cos \left(\psi\left(f\left(\epsilon_{\psi}\right)\right)\right)\right)\right.$.

Скорость быстрых осцилляций по обобщенной координате $x$ как функция медленных компонент примет следующий вид. Для удобства обозначения примем $X\left(f\left(\epsilon_{x}\right)\right)=X$ и $\psi\left(f\left(\epsilon_{\alpha}\right)=\psi\right.$ :

$$
\epsilon_{x}^{\prime}(X, \psi, \tau)=\int-\cos (2 \tau)\left(\beta_{1} X+\beta_{2} \cos (\psi)\right) d \tau
$$

Принимая во внимание уравнения движения по каждой из обобщенных координат, полная кинетическая энергия быстрых осцилляций примет вид

$$
\begin{aligned}
& \frac{1}{2}\left(\dot{\epsilon}^{\prime}{ }_{x}(X, \psi, \tau)^{2}+\dot{\epsilon}_{y}^{\prime}(Y, \psi, \tau)^{2}+\dot{\epsilon}_{\psi}^{\prime}(X, Y, \psi, \tau)^{2}\right) \\
& =\sum_{i=1}^{3} \frac{\epsilon_{i}(Q, \tau)}{2} .
\end{aligned}
$$

Обозначенная функция $(f(\epsilon(x, y, \psi)))$, характеризующая влияние быстроосциллирующих компонент на секулярное движение, определяется усреднением кинетической энергии быстрых осцилляций. Настоящая функция, являющаяся эффективным потенциалом, принимает описанный в (11) вид

$$
\Phi=\frac{1}{2 \pi} \sum_{i=1}^{3} \int_{0}^{2 \pi} \frac{\left(\epsilon_{i}^{\prime}(Q, \tau)\right)^{2}}{2} d \tau .
$$

Подставив обобщенные кооринаты „центр масс-угол“, эффективная потенциальная энергия примет конечный вид

$$
\begin{aligned}
& \Phi(X, Y, \psi)=\frac{1}{16}\left[\left(\beta_{1} X+\beta_{2} \cos (\psi)^{2}\right)+\left(\beta_{1} Y\right.\right. \\
& \left.\left.+\beta_{2} \sin (\psi)^{2}\right)+\left(\beta_{3}(X \sin (\psi)+Y \cos (\psi))+\beta_{4} \sin (2 \psi)\right)^{2}\right] .
\end{aligned}
$$

Для последующего удобства рассмотрения введем безразмерные сферические координаты, где радиус $R$ нормирован на радиус радиочастотной ловушки

$$
X\left[r_{0}\right]=\frac{R}{r_{0}} \sin \phi, \quad Y\left[r_{0}\right]=\frac{R}{r_{0}} \cos \phi, \quad \psi=\psi .
$$

Тогда выражение эффективного потенциала в сферических координатах примет вид

$$
\begin{aligned}
\Phi(R, \phi, \psi)= & R^{2} \beta_{1}^{2}+2 R \beta_{1} \beta_{2} \cos (\phi-\psi) \\
& +\left(R \beta_{3} \sin (\phi+\psi)+\beta_{4} \sin (2 \psi)\right)^{2}
\end{aligned}
$$

\section{Список литературы}

[1] Boyarkin O.V., Kopysov V. // Rev. Sci. Instrum. 2014. Vol. 85. N 3. P. 033106.

[2] Mihalcea B.M., Giurgiu L.C., Stan C., Vişan G.T., Ganciu M., Filinov V., Lapitsky D., Deputatova L., Syrovatka R. // J. Appl. Phys. 2016. Vol. 119. N 11. P. 114303.

[3] Marouane Salhi, Ali Passian, George Siopsis // Phys. Rev. A. 2015. Vol. 95. N 3. P. 033416.

[4] Jorge I., Casas E.M., Villar M., Ortega-Pérez I., LópezFerrer D., Martínez-Ruiz A., Carrera M., Marina A., Martinez P., Serrano H. et al. // J. Mass Spectrometry. 2007. Vol. 42. N 11. P. 1391-1403.

[5] Marshall D.L., Saville J.T., Maccarone A.T., Ailuri R., Kelso M.J., Mitchell T.W., Blanksby S.J. // Rapid Communication in Mass Spectrometry. 2016. Vol. 30. N 21. P. 2351-2359.

[6] Lützow P., Schnell M., Meijer G. // Phys. Rev. A. 2008. Vol. 77. N 6. P. 063402.

[7] Phillips E.S., Hendricks R.J., Abdulla A.M., Ohadi H., Crick D.R., Koo K., Segal D.M., Thompson R.C. // Phys. Rev. A. 2008. Vol. 78. N 3. P. 032307.

[8] Rozhdestvenskii Yu.V., Rudyi S.S. // Tech. Phys. Lett. 2017. Vol. 43. N 8. P. 748-752.

[9] Extensuin of the method of direct separation of motions for problems of oscillatong action pn dynamical systems. Procedia IUTAM, Vol. 19. P. 75-82. 2016. IUTAM Symposium Analytical Methods in Nonlinear Dynamics.

[10] Nagornykh P., Coppock J.E., Kane B.E. // Appl. Phys. Lett. 2015. Vol. 106. N 24. P. 244102.

[11] Alda I., Berthelot J., Rica R.A., Quidant R. // Appl. Phys. Lett. 2016. Vol. 109. N 16. P. 163105.

[12] Alvarez E.J., Brodbelt J.S. // J. Mass Spectrometry. 1995. Vol. 30. N 4. P. 625-631.

[13] Mihalcea B.M., Vişan G.G. // Phys. Scripta. 2010. Vol. 140. P. 014057.

[14] Shah K., Ramachandran H. // Phys. Plasmas. 2009. Vol. 16. N 6. P. 062307. 